Especially valuable is the existence of a voice recording for family and friends in bereavement.

\section{P57 DEVELOPING AN ORAL HISTORY SERVICE IN A HOSPICE}

Clare Williams, Judith Park. St Luke's Hospice, Sheffield, UK

\subsection{6/bmjspcare-2013-000591.79}

Background/context This poster describes the development and implementation of an oral history service at St Luke's Hospice in Sheffield. Oral history involves an interviewer questioning and recording an interviewee. The service was modelled on a successful service at a local palliative care centre.

Aim Patients using the hospice's day centre and in-patient unit would be offered the opportunity to make an audio recording of their life story. Patients would receive a copy of their story on $\mathrm{CD}$ and extra copies would be available to their family and friends with the patient's agreement.

Approach used The service started with a pilot where two patients took part in an interview and then gave feedback. This was positive and so the service was launched. Initially, a member of staff who was a trained oral historian conducted the interviews, but demand for the service led to 20 volunteers being recruited to carry out the interviews. Volunteers were trained in areas such as interview technique, use of recording equipment, ethics, and working with seriously ill patients.

Outcomes To date, 29 patients have used the service and 48 interviews have been recorded. Some patients have used the service to record final messages for family, and one has made a 'Desert Island Discs' style recording complete with music. Others have produced photo books to accompany recordings. The hospice is now able to offer the service to patients looked after at home by our Community nurses. It continues to receive positive feedback from patients and their relatives.

Application to hospice practice The service's model could be used by other hospices to set up their own oral history service. The hospice is also participating in a research study which aims to provide empirical evidence as to the benefits of oral history in palliative care.

\section{P58 REAL WORLD HOSPICE RESEARCH: CHALLENGES AND OPPORTUNITIES}

Sarah Russell, Sharon Chadwick, Kate Hodell, Miller Barbara, Erzsi Nmeth, Ros Taylor, Kimberley McLaughlin, Cate Woodwark. Hospice of St Francis, Berkhamsted, UK

\subsection{6/bmjspcare-2013-000591.80}

Compassionate evidence-based practice, collecting data and building evidence is fundamental to hospice care. The Hospice of St Francis research strategy provides a real world pragmatic effective approach to research evidenced based care in a medium sized hospice.

Challenges

1. Research governance and credibility as a small organisation

2. Time to reflect, discuss and put into practice evidenced based care

3. Being a Researcher Practitioner in the workplace

4. Realistic research with Universities and other research organisations.

5. Using research to disseminate and influence care beyond the hospice walls
Solutions and Opportunities

1. Research strategy, governance and register of all research, audit and service evaluation activity reported to Clinical Governance and Clinical Leads forum.

2. Collaborative research and governance with Institutes of Higher Education and individual researchers wanting to access our hospice as a research site.

3. Research Interest Forum: 2 monthly multi professional forum to discuss issues as researchers in practice and evidence based practice clinicians.

4. Journal Club: Quarterly forum to discuss articles of interest and relevance to practice

5. Think Tank: Monthly multi professional forum to share feedback from conferences and practice initiatives.

6. Case and Care Reflections: monthly forums reflecting on clinical care.

7. Clinical Nurse Specialist Continuous Professional Development Programme: 2 monthly forum delivered by Associate Specialist in palliative care.

8. Schwartz rounds to stimulating discussion and reflection about care

9. Publications and dissemination strategy.

10. A shared passion for real world research.

"Hospices must meet a range of challenges: collecting better data, developing a better understanding of those who needs will dominate future decades, and establishing a robust evidence base for new services" (Calanzani 2013). The Hospice of St Francis whole system approach to real world research makes a difference to care and offers possibilites for other similar sized organisations

\section{P59 ENTER - ENABLING NURSES TO ENGAGE IN RESEARCH: DEVELOPING A CULTURE OF ENQUIRY IN CLINICAL ENVIRONMENTS}

${ }^{1}$ Debra Broadhurst, ${ }^{1,2}$ Jayne Brown. ' LOROS, Leicester, England, ${ }^{2}$ DMU Leicester England

\subsection{6/bmjspcare-2013-000591.81}

Background Identifying the importance of research in end of life care and fostering a culture of enquiry is a key step for healthcare providers if they are to move forward in addressing some of the challenges highlighted by the End of Life Care Strategy (DH, 2008). The ENTER project recognises the need to enhance the ability and confidence of nurses to engage in research activity and view it as an integral part of clinical practice to ensure high quality care at life's end.

Aims

- Empowerment of senior nurses to guide their teams on research processes

- Establish a network of support across the region

- Develop a web-based resource

- Encourage clinical teams to embed a research culture in their working environments

Approach used Seven senior nurses (band 6 and above) attended a three day workshop which comprised of a mixture of taught sessions on the research process, sharing examples of research practices, introducing the 'human face' of teams such as Research and Development and Ethics Committees and reveiwing currently available resources.

Outcomes The group of nurses quickly established themselves as a support network for each other and although the project was originally time limited, a Community of Practice is being 